\title{
Un sistema recomendador móvil de rutinas de ejercicio basado en el perfil del usuario
}

\author{
Jaime Guzmán-Luna ${ }^{1}$, Ingrid-Durley Torres ${ }^{2}$, Sebastián Vallejo² \\ ${ }^{1}$ Universidad Nacional de Colombia, Medellín, \\ Colombia \\ ${ }^{2}$ Institución Universitaria Salazar y Herrera, Medellín, \\ Colombia \\ jaguzman@unal.edu.co, i.torresdiush.edu.co, \\ j.vallejo@iush.edu.co
}

\begin{abstract}
Resumen. Este artículo, describe la investigación y el desarrollo de un sistema recomendador móvil que usando técnicas de Inteligencia Artificial (IA), permite sugerir rutinas de ejercicio, orientadas a fortalecer la calidad de vida del usuario basándose en su perfil antropomórfico y patológico.
\end{abstract}

Palabras clave: rutinas de ejercicio, recomendador, dispositivo móvil, perfil antropomórfico, patología.

\section{Introducción}

Los gimnasios públicos al aire libre, surgen como respuesta al agitado ritmo de vida actual y tienen como objetivo ayudar a conformar sociedades en las que se tenga consciencia acerca del riesgo de una vida sedentaria sobre la salud, a su vez que facilitan adquirir hábitos saludables. Dichos gimnasios, por la flexibilidad de espacio y tiempo, carecen de un asesor experto que oriente a los asistentes en su actividad física y la conveniencia o limitante de uso de las mismas máquinas o de los ejercicios sobre ellas. La diversidad usuarios en edades, peso y la presencia de enfermedades, exigen una clara orientación para los asistentes, cuando acuden a realizar el ejercicio físico, ya que no todas las máquinas resultan convenientes a todos los usuarios, o no todos los ejercicios sobre una misma máquina están recomendados para todos los asistentes.

Ante este panorama, el problema de mejorar la calidad de vida de los usuarios que asisten a los gimnasios públicos al aire libre, resultan no solo infructuosos, sino que en algunos casos, también contraproducentes en la vida de ellos mismos. Bajo las anteriores circunstancias, se hace necesario desarrollar una herramienta soportada en los sistemas de software implementados con técnicas de Inteligencia Artificial (IA), que enseñen el uso correcto de las máquinas disponibles en los gimnasios públicos al aire libre; centrándose en la personalización de los usuarios y su preocupación por mejorar su calidad de vida. Dicha personalización, se determina en este trabajo, a partir de un conjunto de características antropomórficas y patológicas del usuario, que 
permitan especificar la conveniencia de uso de las máquinas disponibles y los ejercicios que pueden realizarse sobre las mismas. Así, según los perfiles del usuario, algunas máquinas o ejercicios serán recomendados a determinados usuarios, mientras que para otros perfiles, los ejercicios deben ser adecuados en intensidad, frecuencia, tiempo o tendrá que ser ignorados como parte de su actividad física. El sistema incorpora además, elementos educativos, que constituyen una alternativa para complementar y reforzar el aprendizaje al usuario; indicando claramente la importancia del uso apropiado de la máquina y el ejercicio, cuando se persigue fortalecer la vida sana y saludable del individuo.

Paralelamente, las nuevas tecnologías en el desarrollo de aplicaciones móviles ofrecen los mecanismos necesarios para que un usuario pueda estar en contacto con la información en cualquier momento y lugar, a través de cualquier tipo de dispositivo móvil. Otro aspecto clave de las aplicaciones desarrolladas a través de estas tecnologías, se soporta en la portabilidad y masificación que ofrecen. Finalmente, y debido a su cotidianidad, los usuarios se sentirán en confianza utilizando este tipo de aplicaciones con las que se encuentra familiarizados. Tales características, convierten la tecnología móvil, en una alternativa para proporcionar conocimientos de formación, con mayor facilidad de retención gracias a la disponibilidad de un estudio continuo.

La motivación de este trabajo se concentra en establecer la investigación y el desarrollo de un sistema recomendador móvil que usando técnicas de IA, permita sugerir rutinas de ejercicio, orientadas a mejorar la calidad de vida del usuario, basándose para ello, en su perfil antropomórfico y patológico. Esta motivación es debida, a la falta de un sistema que promueva el ejercicio de la manera apropiada, sobre los elementos disponibles en los gimnasios públicos al aire libre, los cuales nacen como cumplimiento de las obligaciones de las instituciones gubernamentales, basadas en la protección de la vida de los ciudadanos de todas las edades. Lo que significa también, ofrecer espacios adecuados para el cuidado de la salud y para poder realizar actividades físicas de forma cotidiana, fomentando una vida saludable para el individuo y la sociedad.

\section{Estado del arte}

Usados para combatir los índices de sobrepeso y obesidad presentes en la comunidad de Shanghái y Bejín, China, los gimnasios al aire libre comenzaron a ser aplicados hace más de diez años, como menciona [1] en su estudio "La integración de Gimnasios al Aire Libre y el desarrollo local". En España, por su parte, se incorporaron al espacio ciudadano hace aproximadamente nueve años, con el fin de combatir la aparición de los efectos generados por el envejecimiento, cambiando también su denominación a Circuitos Biosaludables o Parques Geriátricos, según explica Hernández [2]. Estos escenarios, están ubicados al aire libre, en zonas verdes, dotados de máquinas diseñadas para soportar la intemperie, y adaptados para realizar los ejercicios que se realizan en los gimnasios comunes, permitiendo realizar trabajos de fuerza, de tipo aeróbico, de coordinación y movilidad articular [3]. Como explican Sáez [4], la base del trabajo que se realiza en estos espacios, está fundamentada en una rama de la fi- 
sioterapia conocida como quinesioterapia o cinesiterapia, la cual se enfoca en el tratamiento de patologías a través del movimiento bien sea pasivo o activo. En Colombia, ciudades como Bogotá, Medellín, Pereira, entre otras, cuentan con estos gimnasios lo largo y ancho de su geografía, generando nuevas posibilidades a la comunidad para la práctica de ejercicio físico.

Si bien se evidencia en los estudios [5], [6], que este tipo de escenarios fue pensado para la población adulta, al estar al alcance de toda la comunidad y no tener ninguna restricción de uso, ni nadie que vigile o acompañe la práctica del ejercicio, la afluencia de usuarios es masiva y de una gran diversidad.

Desde la perspectiva de las aplicaciones móviles, la oferta es aún más variada y de múltiples alcances. La Tabla 1, presenta de manera resumida y por límites de espacios solos algunas de ellas, orientadas específicamente a caracterizar la actividad física en gimnasios, aunque ninguna se oriente a los ubicados al aire libre;

Tabla 1. Aplicaciones móviles asociados a actividad física.

\begin{tabular}{|c|c|c|c|}
\hline $\begin{array}{c}\text { NOMBRE } \\
\text { APLICACIÓN }\end{array}$ & $\begin{array}{c}\text { SISTEMA } \\
\text { OPERATIVO }\end{array}$ & $\begin{array}{c}\text { TIPO DE } \\
\text { EJERCICIO }\end{array}$ & CARACTERISTICAS \\
\hline GYM GUIDE & IOS, ANDROID & Gimnasio & $\begin{array}{l}\text { - Formato en inglés. } \\
\text { - Base de ejercicios. } \\
\text { - Imágenes de ejercicios. }\end{array}$ \\
\hline UFC GYM & ANDROID & Gimnasio & $\begin{array}{l}\text { - GPS para ubicar el gimnasio de entrena- } \\
\text { miento. (horarios) } \\
\text { - Menú en inglés. }\end{array}$ \\
\hline $\begin{array}{l}\text { COMPLETE } \\
\text { GYM GUIDE }\end{array}$ & ANDROID & Gimnasio & $\begin{array}{l}\text { - Idioma inglés. } \\
\text { - Recomendaciones para ejercicio. } \\
\text { - Fotos e imágenes para explicar los ejerci- } \\
\text { cios. }\end{array}$ \\
\hline $\begin{array}{c}\text { EJERCICIOS } \\
\text { GYM }\end{array}$ & ANDROID & Gimnasio & $\begin{array}{l}\text { - Video de los ejercicios con introducción en } \\
\text { Ingles. } \\
\text { - Espacio para diseñar rutina. } \\
\text { - Rutinas recomendadas por objetivos. }\end{array}$ \\
\hline $\begin{array}{l}\text { GYM GUIA } \\
\text { COMPLETA }\end{array}$ & ANDROID & Gimnasio & $\begin{array}{l}\text { - Videos de los ejercicios (como avatar) } \\
\text { - Explicación del ejercicio abajo del video. } \\
\text { - Rutinas por niveles y cantidad de días a la } \\
\text { semana. } \\
\text { - Tips de entrenamiento. } \\
\text { - calculadoras de calorías, IMC entre otras. } \\
\text { - Teoría sobre alimentación. } \\
\text { - Imágenes de motivación. }\end{array}$ \\
\hline GYM SPORT & ANDROID & Gimnasio & $\begin{array}{l}\text { - Imágenes de un libro de entrenamiento. } \\
\text { - Guía de rutinas por objetivo y por nivel. } \\
\text { - Teoría sobre alimentos } \\
\text { - Teoría sobre anatomía y antropometría. }\end{array}$ \\
\hline
\end{tabular}

Sin embargo, se destaca la articulación con hardware del dispositivo, como el GPS, la incorporación de contenido multimedia para presentar la forma en que se debe realizar el ejercicio, el acompañamiento de calculadores para definir el IMC (índice de Masa Corporal), el peso ideal en otros; también se destaca, el idioma de presentación, donde prima el inglés o su carácter gratuito por un limitado tiempo, además de 
la ausencia de consideraciones pedagógicas o patológicas del individuo; sin mencionar que se trata ejercicios físico que deben ser desarrollados en máquinas con especificaciones técnicas acordes con las disponibles en los gimnasios de servicio pago.

Desde el aspecto académico, en la literatura, no existen definiciones formales de rutina de ejercicios; sin embargo, se pueden afirmar de manera categórica que [7] una rutina está compuesta de ejercicios físicos y que estos a su vez, son entendidos como aquellas actividades físicas que son planificadas, estructuradas, con un objetivo definido y con las cuales se pretende mejorar la forma física [8]. Los trabajos, también presentan algunos tipos de sistemas ideados para la recomendación de rutinas, cada uno de ellos proponiendo diferentes métodos o técnicas para hacerlo. A continuación Tabla 2, recopila las técnicas más utilizadas.

Desde esta perspectiva, una de las limitantes más significativa sobre estos sistemas es que exigen tener información previa para evitar el arranque en frío [7], [9]. Otro aspecto preocupante, es la imposibilidad de incorporar dentro de los perfiles, los perfiles patológicos de los usuarios, ya que solo se concentran en recomendar en función de la antropometría del usuario.

Tabla 2. Métodos para la recomendación de rutinas.

\begin{tabular}{|c|c|}
\hline Método & \multicolumn{1}{|c|}{ Descripción } \\
\hline Sistemas de agentes [10] & $\begin{array}{l}\text { Define diferentes agentes encargados de realizar distintas tareas, } \\
\text { el agente principal, es denominado entrenador y es encargado de } \\
\text { considerar el perfil del usuario y su condición para recomendar. }\end{array}$ \\
\hline Sistema experto difuso [11] & $\begin{array}{l}\text { Basado en una serie de reglas que permiten tomar decisiones } \\
\text { como si se tratara de un experto, adquiere retroalimentación del } \\
\text { usuario y usa lógica difusa para cuantificarla. }\end{array}$ \\
\hline Basado en Ecuaciones [13] & $\begin{array}{l}\text { Según una ecuación que calcula el trabajo necesario para con- } \\
\text { sumir una cantidad de calorías, recomienda la (rutina) al usuario. }\end{array}$ \\
\hline Basada en grafos [14] & $\begin{array}{l}\text { El modelo de rutina se representa con grafos, calculando el peso } \\
\text { de los nodos según tiempo y exigencia, elige el grafo que mejor } \\
\text { se ajuste a los requisitos del usuario. }\end{array}$ \\
\hline Índices de similitud [15] & $\begin{array}{l}\text { Almacenan la información de los usuarios, conocimiento sobre } \\
\text { medicina y nutrición en ontologías y con estas alimentan el } \\
\text { sistema a fin de elegir la rutina que mejor se ajuste al usuario. }\end{array}$ \\
\hline
\end{tabular}

\section{Public GYM: arquitectura y funcionalidad}

Public Gym es un sistema recomendador móvil que basado en técnicas de Inteligencia artificial, permite sugerir a un usuario una rutina de ejercicio, específicamente orientada a la actividad física en los gimnasios públicos al aire libre; considerando para ello las características antropométricas y patologías del usuario. Public Gym, es soportado por componentes pedagógicos que van desde la teorías de aprendizaje significativo y constructiva [14], hasta el uso de recursos como los objetos de aprendiza- 
je (OA) [15], los cuales pueden ser visualizados en distintos dispositivos, a fin de aprovechar su accesibilidad, portabilidad y masividad.

\subsection{Descripción de la arquitectura}

Este sistema recomendador, debe basarse en una arquitectura de cómputo en la nube que permita su acceso por parte de los usuarios en cualquier momento y lugar, evitando el gasto en equipo cómputo para el mantenimiento del sistema, considerando a su vez, las limitantes de presentación y almacenamiento de los dispositivos móviles. Por otra parte, se hace necesario que los usuarios puedan registrar su perfil, obtener e instalar la aplicación móvil desarrollada desde el sistema y actualizar su información a través del tiempo, razón por la cual, se incorpora un proceso de autenticación y acceso sin límite de tiempo o veces al usuario.

El sistema, permite además, crear una aplicación educativa representando el conocimiento del orientador o entrenador en deportes, para ello se ha decido implementar un sistema experto que contiene una especificación de reglas que permiten recomendar las rutinas según los principales perfiles de usuario. Para lograr lo señalado, se propone una arquitectura modular de cuatro capas, como la señalada en la Fig. 1.

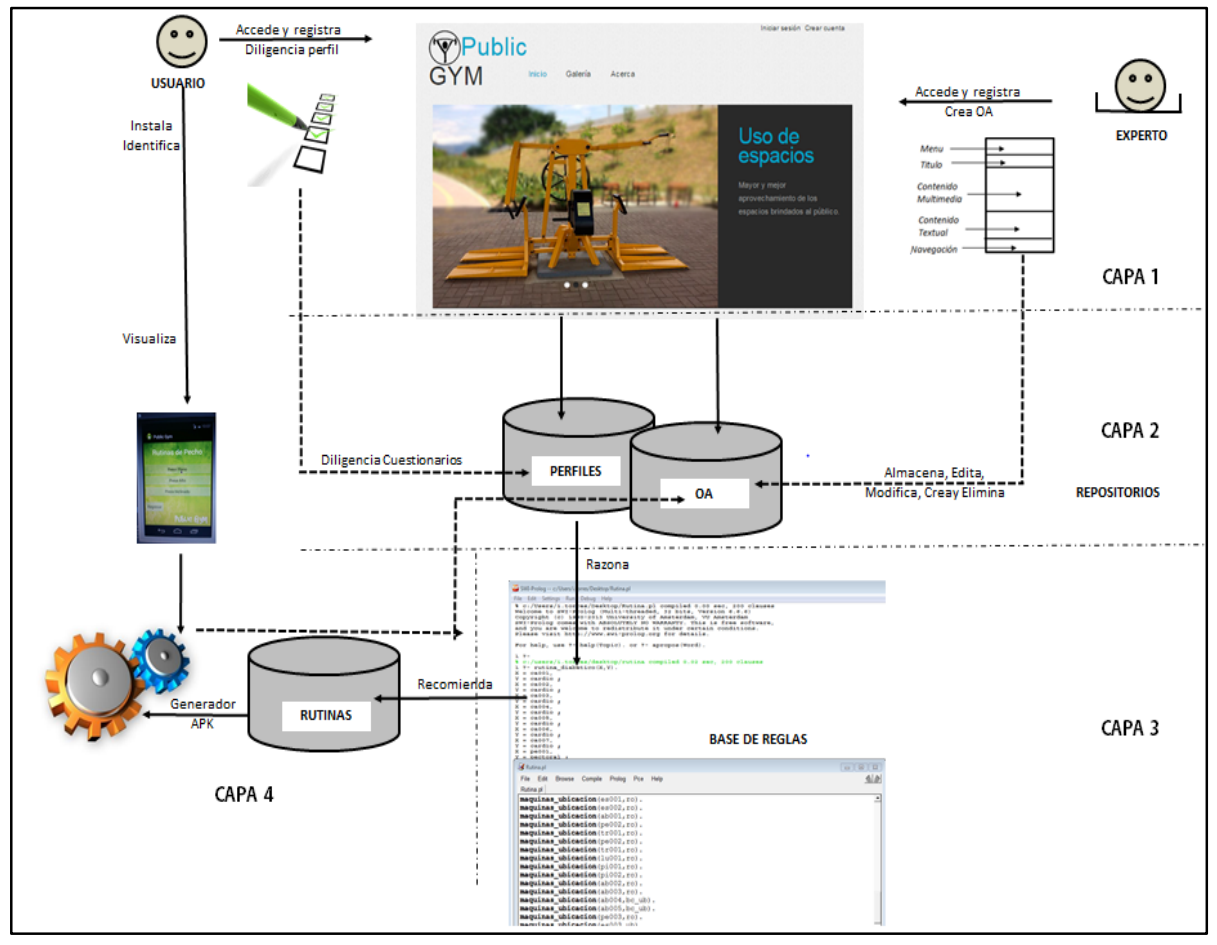

Fig. 1. Arquitectura del sistema recomendador.

En tal arquitectura, se consideran los siguientes Actores: 
- A0: Usuario: Es el encargado de consumir los objetos de aprendizaje suministrados de acuerdo a un perfil establecido a través de una rutina de ejercicios y visualizados desde su dispositivo móvil.

- A1: Experto: Es el encargado de crear, editar, eliminar y actualizar, los contenidos registrados en el sistema recomendador, como son los OA y la información de las máquinas de gimnasio al aire libre. Para ello hace uso de los contendidos digitales (textos, imágenes y videos) y un par de plantillas para disponer tal contenido. Es él quien tiene la responsabilidad de diseñar acorde a su formación los parámetros para establecer las rutinas según los perfiles.

- A2: Administrador: Es el encargado de gestionar el sistema desde su aspecto técnico, además de los otros usuarios. Una de sus tareas claves, está representada en la traducción del conocimiento del experto al modelo de representación de reglas del sistema.

La arquitectura de cuatro capas, consta de una página web, de interfaz inicial, a través de la cual se consigue el acceso a la capa dos, la cual contiene el repositorio de OA, junto con la base de datos de los perfiles de cada uno de los usuarios (información suministrada por los usuarios, cuando se registra). En la tercera capa, se encuentra el sistema de reglas corazón del recomendador, además de la representación de la base de conocimiento del experto humano; en esta tercera capa, también se halla el repositorio de gimnasios, máquinas disponibles y actividad muscular permitida en cada una de ellas. En la capa final, se halla el componente responsable de seleccionar la rutina específica recomendada para el perfil de un usuario. Esta última capa, es accedida, desde la aplicación instalada en el dispositivo móvil, otorgando la portabilidad al recomendador. Las tres primeras capas, también están habilitadas para el usuario experto, quien las accede para actualizar el repositorio de objetos de aprendizaje, incorporado nuevos ejercicios, máquinas o la disponibilidad de un nuevo gimnasio.

\subsection{Funcionalidad de la arquitectura}

El sistema opera de la siguiente manera: Primero, un usuario experto, registra los OA, para ello, se hace uso de dos plantillas. Las rutinas, también son construidas bajo las recomendaciones del experto, y constituyen una secuencia de OA, acompañadas del trabajo del administrador, quien las convierte en conocimiento comprensible por el software. Una vez construido, el sistema de conocimiento, ya puede ser accedido por un usuario asistente a un gimnasio público, quien ingresa (normalmente desde su equipo de cómputo), a la página de acceso de la aplicación publicgym, generando un usuario y una contraseña; Posteriormente, el usuario debe diligenciar un cuestionario, el cual brinda la información necesaria al sistema para procesar mediante su base de reglas (sistema experto), la clasificación del usuario, dentro de uno de los perfiles disponibles (los cuales serán descritos en el apartado siguiente). El perfil del usuario es almacenado, junto con la identificación de la rutina que le corresponde. Una vez, el usuario lo desee, podrá acceder desde su dispositivo móvil, instalar la aplicación y luego de autenticarse, el sistema le habilitará la descarga de su correspondiente rutina. Esto con el fin de ahorrar espacio de almacenamiento, evitando otros elementos inne- 
cesarios. Las cuatro capas, numeradas en la Fig. 1, están dispuestas en la nube, a fin de que brinden disponibilidad y acceso libre al usuario, ocupándose el sistema de todos los procesos de cómputo.

\subsection{Gimnasios y máquinas}

Para identificar cada uno de los gimnasios públicos existentes, se realizó primero, un levantamiento de información directo, que exigía visitar la página web de los entes gubernamentales, a fin de revisar sus registros públicos al respecto. Esta información fue contrastada con el "voz a voz" de distintos usuarios, quienes aportaron información sobre la ubicación de algunos gimnasios, ayudando a construir nuestro registro final el cual, se generó, como un consolidado con toda la información que relacionaba la dirección exacta de ubicación de cada gimnasio. Con el ánimo de dar un acercamiento real, se incorporó información acerca de qué máquinas había y la cantidad y el tipo de cada una de ellas en cada gimnasio. Finalmente y para dar un mayor detalle, se construyó un consolidado, cruzando cada máquina disponible, versus la zona muscular que permite ejercitar. El consolidado ha sido recopilado en una taba, de la cual se muestra una porción en la Fig. 2.

\begin{tabular}{|c|c|c|c|c|c|}
\hline \multicolumn{4}{|c|}{ CODIGOS EJERCICIOS } & \multicolumn{2}{|c|}{ Tabla referencias Zona ejercicio } \\
\hline NOMBRE EJERCICIO & MAQUINA & EJERCICIO & UBICACIÓN & CA & Cardio \\
\hline Aero Sky & 1 & CA001 & $\mathrm{RO}$ & $\mathrm{PE}$ & Pectoral \\
\hline Elíptica & 2 & CA002 & RO & HE & Hombro, Trapecio \\
\hline Spinning & 3 & CA003 & RO & ES & Espalda \\
\hline Escalador & 4 & CA004 & RO & TR & Triceps \\
\hline Jinete & 5 & CA005 & $\mathrm{BC}-\mathrm{RO}$ & BI & Biceps \\
\hline Pedaleo & 6 & CA006 & RO & AB & Abdomen \\
\hline Mesa Cardio & 7 & CA007 & RO & LU & Lumbares \\
\hline Press de Pecho en Mesa Cardio & 7 & PE001 & RO & PI & Muslo, Pierna, Gluteo \\
\hline Press de Hombro en Mesa de Cardio & 7 & HE001 & RO & & \\
\hline SKY & 8 & CA008 & $\mathrm{BC}$ & \multicolumn{2}{|c|}{ Tabla referencias ubicación } \\
\hline Pendúlo & 9 & CA009 & $\mathrm{BC}$ & RO & Robledo \\
\hline Dominadas Agarre Abierto & 10 & ESO01 & RO & BC & Barrio Cristobal \\
\hline Dominadas Agarre Cerrado & 10 & ES002 & RO & UB & Unidad Deprotiva Belen \\
\hline Elevación de piernas en paralelas & 10 & AB001 & RO & & \\
\hline $\begin{array}{l}\text { Fondos en paralelas } \\
\end{array}$ & 10 & PE002 & RO & & \\
\hline Fondos en paralelas & 10 & TR001 & $\mathrm{RO}$ & & \\
\hline Fondos en paralelas & 11 & PE002 & RO & & \\
\hline Fondos en paralelas & 11 & TR001 & $\mathrm{RO}$ & & \\
\hline Hiperextensiones & 11 & LU001 & RO & & \\
\hline Press de Piernas & 12 & PI001 & RO & & \\
\hline Press de Pierna Individual & 12 & P1002 & RO & & \\
\hline Elevación de Piernas en Banco plano & 13 & AB002 & RO & & \\
\hline Crunch en banco Plano & 13 & AB003 & RO & & \\
\hline Crunch en banco inclinado & 14 & AB004 & $B C-U B$ & & \\
\hline Elevación de Piernas en Banco Inclinado & 14 & ABO05 & $B C-U B$ & & \\
\hline Press de Pecho en Maquina & 15 & PEO03 & RO & & \\
\hline Remo en Maquina & 16 & ES003 & UB & & \\
\hline Press Plano & 17 & PE004 & $\mathrm{BC}-\mathrm{RO}$ & & \\
\hline
\end{tabular}

Fig. 2. Información de Gimnasios, Máquinas y zona muscular.

\subsection{Objetos de aprendizaje (OA)}

Los OA son unidades de estudio, ejercicios o prácticas que pueden ser consumidas en una sección sencilla y que representan gránulos reutilizables que pueden ser creados, sin importar qué tipo de medio de entrega será utilizado [16]. Idealmente, los OA 
pueden ser reutilizados y conectados juntos para construir aplicaciones que estén destinadas a servir a un determinado propósito o meta. En consecuencia, los OA necesitan ser libres del entorno, lo que significa que tienen que llevar información útil que describa el tipo y el contexto en el que pueden ser usados [17]. El diseño entonces de los OA, está básicamente representado por un par de plantillas, sobre las cuales se va a recopilar la información pedagógica [18]. Una de ellas, está diseñada para presentar el contenido educativo-motivacional (Fig. 3 A.), el cual corresponde a información clave que indica al usuario la razón por la cual se clasificó en ese perfil, o porque resulta tan importante que él realice los ejercicios bajo los parámetros que su rutina presenta. La segunda, corresponde al aspecto educativo-técnico (Fig. 3 B.), en donde se describe de manera textual y con un corto video, la manera correcta de realizar ese ejercicio y la forma adecuada de adoptar una buena postura sobre la máquina. El conjunto ordenado de OA, bajo determinados criterios definidos por los perfiles, responden a la especificación de una rutina de ejercicio. Tal como se señala en la Fig. 3. Cada OA, es almacenado dentro del repositorio, bajo los metadatos del estándar LOM [16]. Con ello, se facilita su clasificación, almacenamiento y recuperación.

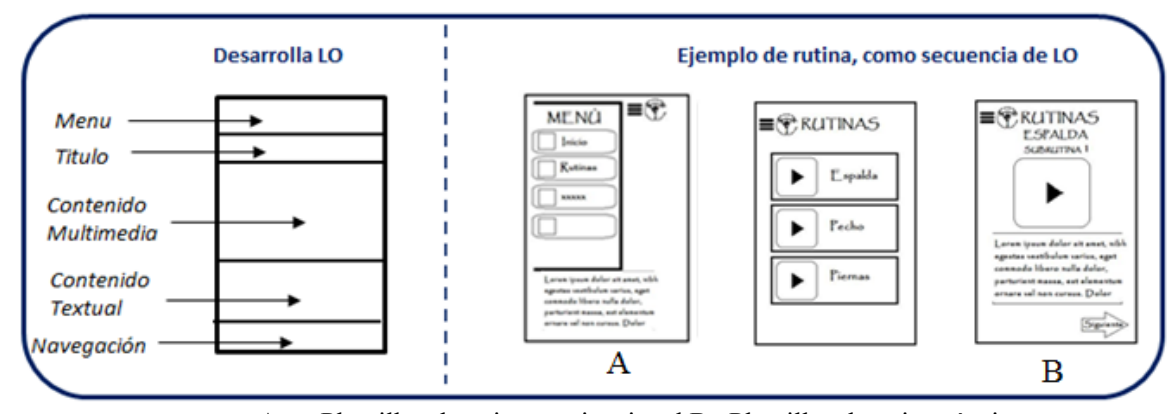

A. Plantilla educativo-motivacional B. Plantilla educativa-técnica

Fig. 3. Plantilla de OA.

\subsection{Perfiles de usuario}

Para identificar la población que asiste a los gimnasios públicos [19], se consolidaron datos mediante el modelo de encuesta, sobre una muestra de 502 usuarios. Las variables que se recogieron, registran formas cualitativas y cuantitativas, definidas desde aspectos antropométricos como: edad, peso y talla, de los usuarios que asisten a los gimnasios públicos. Con tales datos, se pudo determinar el IMC o Índice de Quetelet [8], además de la frecuencia cardiaca máxima del sujeto. Las variables también, incluyen datos sobre el estado actual de salud de los usuarios, considerando la presencia y ausencia de enfermedades; finalmente, se ahondó en aspectos propios del entrenamiento como la hidratación antes, durante y después del ejercicio, la duración, intensidad y frecuencia de la sesión de entrenamiento a la que ellos estaban familiarizados realizar. Se incluyeron también, cuestionamientos relacionados con el nivel educativo, el grado económico e incluso la tenencia de dispositivos móviles. Los datos recogidos en las encuestas, fueron analizados con la ayuda del software estadístico de 
acceso libre R versión 3.0.2, que permiten identificar las variables de mayor relevancia y facilitan la caracterización de la población que fue sujeto de estudio. Para este análisis se utilizó una estadística descriptiva en la que se usaron medidas de tendencia central que corresponden a valores numéricos que ayudan a localizar de alguna manera el centro del conjunto de datos. Para ello se manejó una media aritmética, que es aplicada estadísticamente para calcular datos que no se encuentran agrupados. Para determinar su valor sumamos cada uno de los datos y lo dividimos por el número de valores de la muestra de la siguiente manera:

$$
\overline{\mathrm{X}}=\frac{\sum_{i=1}^{n} X i}{n}=\frac{X_{1}+X_{2}+X_{3}+\ldots++X_{1}}{n}
$$

donde: $\overline{\mathrm{X}}$ : Es la media calculada

$\mathrm{n}$ : Número de datos

$\mathrm{x}_{\mathrm{i}}$ : Es cada uno de los datos de la muestra

Luego de valorar y clasificar los usuarios bajo varios parámetros de las variables antes citadas, se consolidó la información relacionada con el hallazgo de personas con presencia de enfermedades crónicas, como hipertensión y diabetes Mellitus. Este estudio, se acompañó de la identificación del tipo de ejercicio que estos sujetos realizan teniendo en cuenta su condición y estado de salud. Él estudió permitió identificar desde las respuestas que un total de 16 personas con diabetes, 23 con alteraciones de la presión sanguínea, 2 con alguna patología que preferiblemente no mencionaron y 21 que reconocen su situación de sobrepeso, dato que difiere mucho de la realidad encontrada por cálculo de IMC. Los restantes 438, encuestados manifestaron no presentar ningún tipo de enfermedad, - clasificados como sanos-. Con la selección de la muestra y el resultado del estudio, se encontró que de los pacientes con diabetes son equivalentes al 3,2\% de la muestra, un 4,6\% son hipertensos, el 9,4\% registra sobrepeso (dentro de los que está el 1,8\% que registra sobre peso), el otro $82,4 \%$ clasificó como saludable. Dados los análisis de frecuencia de asistencia a los gimnasios, se incluyó el perfil, el sedentario, el cual está representado por un $8,3 \%$ de la población.

\subsection{Recomendador móvil}

Es importante recordar que el propósito de las representaciones que se producen en un sistema de recomendación, se reducen a ayudar a los seres humanos a tomar decisiones y descubrir nuevos elementos, con menos esfuerzo, que si realizaran la actividad de manera manual. Considerando lo expuesto, resulta conveniente incorporar técnicas de IA, que basadas en conocimiento permitan explorar la estructura y las relaciones del mundo o dominio al que pertenece el problema, de la misma forma que permiten la reducción del número de posibilidades, tal como hacen los humanos. Las técnicas de recomendación [13] poseen varias clasificaciones basándose en las fuentes de datos sobre las cuales se hacen las recomendaciones y el uso que se le da a estos datos. La técnica de recomendación basada en el conocimiento (KBR: KnowledgeBased Recommendation) intenta sugerir objetos, basados en inferencias sobre las preferencias y necesidades del usuario. Se distingue de las demás técnicas, en que ésta 
tiene un conocimiento previo funcional sobre cómo un ítem en particular puede satisfacer la necesidad de un usuario y por tanto puede razonar sobre la relación entre esta necesidad y una posible recomendación. El perfil del usuario, puede ser cualquier estructura de conocimiento que soporte esta inferencia.

El sistema recomendador trabaja en función de la interpretación de la información suministrada por un usuario. En este caso, se ubica al individuo dentro de un grupo poblacional y se le asigna una rutina que posteriormente podrá seguir a través de su visualización en un dispositivo móvil. Se trata entonces, de recomendar una rutina, basada en el conocimiento de un experto y la descripción del perfil del usuario, adicionándolo a un grupo poblacional identificado por las patologías más comunes. Con la información anterior, se construye un sistema de reglas que permite razonar y recomendar la rutina que resulte más conveniente, La Fig. 4, señala apartes de la base de conocimiento y la rutina recomendada para un diabético, bajo prolog. En este caso la rutina para el diabético, está adaptada de acuerdo a dos características principales que son: la zona de aplicación de la insulina (insulinodependientes) y la frecuencia del ejercicio.

Las rutinas que el sistema diseñe se ubican bajo la modalidad de circuitos y tendrán una base de 8 ejercicios de fuerza resistencia (FR) y 1 ejercicio aeróbico. Los 8 ejercicios de FR serán seleccionados teniendo en cuenta la zona de aplicación de la insulina en los pacientes. El sistema puede seleccionar los 8 ejercicios aleatoriamente teniendo en cuenta que un grupo muscular no este seguido de un ejercicio para la misma zona, es decir, en el Circuito se trabajaran los grupos musculares aproximadamente 2 veces por serie pero no de manera continua; así: 1 ejercicio pecho, 1 ejercicio de espalda, 1 ejercicio de brazos, 1 ejercicio de muslo/pierna, 1 ejercicio de abdomen, 1 ejercicio de hombro, 1 ejercicio de pecho, 1 ejercicio de espalda.

\begin{tabular}{|c|c|}
\hline $\begin{array}{l}\text { ejercita(tr003, triceps). } \\
\text { ejercita (tr004, triceps). } \\
\text { ejercita (pi009, muslo_pierna_gluteo). } \\
\text { ejercita (pi010, muslo_pierna_gluteo). } \\
\text { ejercita (es008, espalda). } \\
\text { ejercita (tr005, triceps). } \\
\text { ejercita (bi001, biceps). } \\
\text { ejercita (he005, hombro_trapecio). } \\
\text { sperfil usuario } \\
\text { perfil (usuario, diabetico). } \\
\text { perfil (usuario, hipertenso). } \\
\text { perfil (usuario, obeso). } \\
\text { perfil (usuario, sano). } \\
\text { perfil (usuario, sobrepeso). }\end{array}$ & 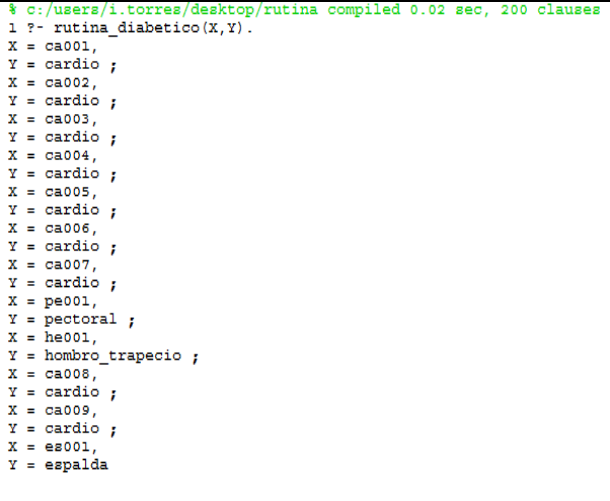 \\
\hline
\end{tabular}

Fig. 4. Base de Conocimiento Vs. Rutina recomendada para un diabético.

Dado que la zona de aplicación de la insulina no podrá ser trabajada, el sistema detectara tras la indicación del usuario dicha zona, y deberá eliminar el ejercicio que se enfoca en ese trabajo y cambiarlo por otro distante a dicha zona. Ejemplo: si en la rutina anterior el usuario indicó que la zona de aplicación fue el abdomen, este ejerci- 
cio será omitido y se adicionara uno que será del tren inferior ya que para esta zona existe solo un ejercicio.

Toda esta información es modelada bajo reglas, tal como la mostrada en la Fig. 5. Aunque, en Fig. 5, solo se muestra una de las reglas del perfil diabético, el sistema también permite filtrar las máquinas según la ubicación del usuario y ejercicios según el perfil, definiendo intensidad y frecuencia. Evitando recomendar ejercicios no convenientes al usuario.

rutina_diabetico $(W, D):-$ perfil (usuario,diabetico), zona_inyeccion_diabetico $(Z)$, ejercita $(W, D)$, not $(D=Z)$.

Fig. 5. Regla base para recomendar rutina de diabético.

Las rutinas son almacenadas en un repositorio como archivos XML, que direccionan las URL de cada OA. Esto facilita su conversión a archivos APK y alivianan la tarea de despliegue del video dentro del archivo, el cual finalmente es visualizado por cada usuario en su dispositivo móvil, tal como lo muestra la Fig. 6.

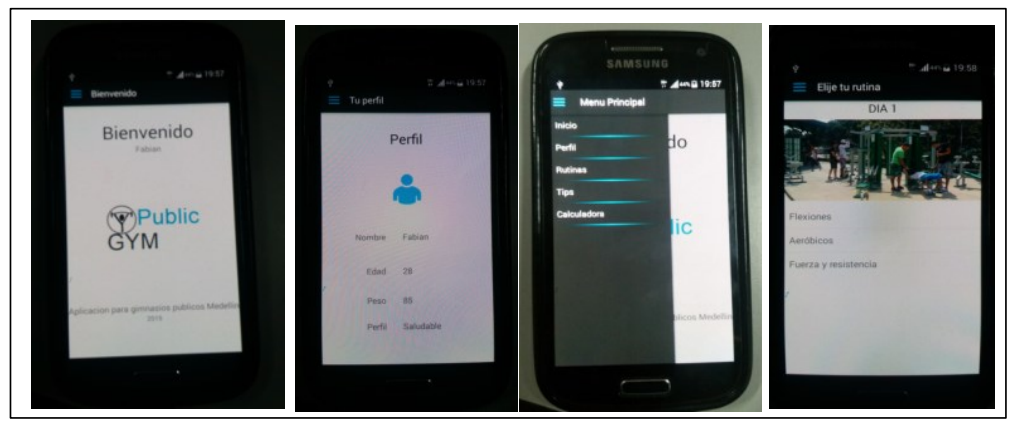

Fig. 6. Aplicación en dispositivo móvil.

\section{Conclusiones}

Este trabajo presenta las bases para el desarrollo de un sistema recomendador móvil, que usando técnicas de IA, permite sugerir rutinas de ejercicio, orientados a fortalecer la calidad de vida del usuario, basándose en su perfil antropométrico y patológico. La arquitectura de PublicGym, proporciona los niveles necesarios para el diseño de OA y la generación de rutinas, como secuencias de los mismos; mientras el sistema de reglas, permite recomendar una rutina basado en un perfil de un usuario, el cual es resultado de la combinación de las características antropométricas, junto con las patológicas. La arquitectura de cómputo en la nube, permite el acceso libre a la aplicación, así como libera al usuario de la carga computacional del proceso de recomendación y de la visualización de los videos, en el dispositivo móvil.

Mediante cada rutina recomendada, se espera el usuario ejecute de manera correcta su actividad fisica, mejorando su calidad de vida, razón por la cual, el recomendador incluye aspectos pedagógicos claves como la motivación y la técnica del ejercicio sobre la correspondiente máquina. 
Como trabajo futuro, se espera incorporar técnicas de planificación en IA, para construir de manera dinámica las rutinas de ejercicio, incorporando la posibilidad de considerar algunas preferencias del usuario.

Agradecimientos. Los autores agradecen a Colciencias, a la Institución Universitaria Salazar y Herrera y a la Universidad Nacional de Colombia por cofinanciar el proyecto de investigación "Desarrollo de una plataforma tecnológica para la publicación de objetos de aprendizaje personalizados, aplicados al uso adecuado de los gimnasios al aire libre, en dispositivos móviles", presentado y aprobado en la convocatoria 626 del año 2013.

\section{Referencias}

1. Arufe V, Cortés L, Suárez X.: Estudio descriptivo de los servicios ofrecidos para los usuarios de parques biosaludables de Galicia. Retos, nuevas tendencias en educación física, deporte y recreación, no. 24, pp. 60-62 (2013)

2. Hernández E.: Estudio de los circuitos biosaludables para la tercera edad en España. Revista Internacional de Medicina y Ciencias de la Actividad Física y el Deporte, vol. 9, pp. 25-38 (2009)

3. Donciu M., Loniţă M., Dascălu M., Trăuşan-Matu E.: The Runner - Recommender System of Workout and Nutrition for Runners In: Proc. 13th Int. S Symbolic and Numeric Algorithms for Scientific Computing (SYNASC), pp. 230-238 (2012)

4. Sáez C, Rodríguez C., López R.: El parque geriátrico: Fisioterapia para nuestros mayores. Gerokomos, vol. 18, no.2, pp.84-88 (2007)

5. Hernández E, Fernández Rodríguez E, Merino Marbán R, Chinchilla Minguet L: Análisis de los circuitos biosaluables para la tercera edad en la provincia de Málaga (España). Retos, Nuevas Tendencias en Educación Física, Deporte y Recreación, vol. 17, pp. 99-102 (2010)

6. Pérez R, García Soidán J, Chinchilla Minguet J: Circuitos biosaludables y cumplimiento de las recomendaciones sobre actividad física para mayores. Revista Internacional de Medicina y Ciencias de la Actividad Física y el Deporte, vol. 12, no. 47, pp. 445-458 (2011)

7. Böhmer M., and Bauer G.: Exploring the Design Space of Context Aware Recommender Systems that Suggest Mobile Applications. In: CARS-2010, September 26, Barcelona, Spain, (2010)

8. American College of Sports Medicine (ed.): Manual ACSM para la valoración y prescripción del ejercicio, Paidotribo, Badalona (2014)

9. Setten M., Pokraev S., Koolwaaij J.: Context-Aware Recommendations in the Mobile Tourist Application COMPASS. Adaptive Hypermedia and Adaptive Web-Based Systems. Lecture Notes in Computer Science, Volume 3137, Springer-Verlag, pp. 235-244 (2004)

10. Barrera L., Ramos A. C., Barraza A., Martinez S.: ZuRoutine: Perzonalized Model of Exercise Routines. In: 6th Computer Colombian Congress, CCC 2011, pp. 1-6 (2011)

11. Suh M. K., Lee K., Nahapetian A., Sarrafzadeh M.: Interval Training Guidance System with Music and Wireless Group Exercise Motivations. In: International Symposium on Industrial Embedded Systems, SIES, pp. 110-119 (2009)

12. Palomares R. A., Ramírez J., Montaño A., Navarro J. A., Vazquez J. L: The Intelligent Personal Trainer. In: Proc. 16th IEEE International Conference on Electronics, Communications, and Computers (CONIELECOMP), no. 49 (2006) 
13. Terveen, L., Hill, W.: Beyond Recomendador Systems: Heling People Help Each Other. In: J. M. Carroll (ed.) Human-Computer Interaction in the New Millennium, AddisonWesley, ACM Press, New York, ch. 22, pp. 487-509 (2001)

14. Lim J. E., Choi O. H., Na H. S., Baik D. K.: A Context-Aware Fitness Guide System For Exercise Optimization In U-Health. IEEE Trans. Inf. Technol. Biomed., vol. 13, no. 3, pp. 370-379 (2006)

15. Wiley, D. A.: Connecting Learning Objects to Instructional Design Theory: A Definition, A Metaphor, A Taxonomy. Utah State University (2002)

16. IEEE Standards Department (2002). Draft Standard for Learning Object Metadata. IEEE Publication P1484.12.1/D6.4 (2002)

17. Vossen, G. P., Westerkamp G.: UDDI for E-Learning: A Repository for Distributed Learning Objects. In: Proc. 2nd International Conference on Information and Knowledge Sharing (IKS2003), Scottsdale, AZ, USA, pp. 101-106 (2003)

18. Ausubel, D., Novak, J., Hanesian, H.: Introducción a la Investigación Pedagógica. 3rd edn. Interamericana, Mexico (1989)

19. Pérez R, García Soidán J, García Núñez F, Chinchilla Minguet J.: Los parques biosaludables en Galicia. Revista de Investigación en Educación, vol. 8, pp. 55-61 (2010) 